\title{
Efecto de la Trayectoria Académica en el Desempeño de Estudiantes de Ingeniería en Evaluaciones Nacionales
}

\author{
Jaime H. Martínez-Padilla y Jorge A. Pérez-González \\ Universidad Autónoma de San Luis Potosí, Facultad de Ingeniería, Av. Dr. Manuel Nava No 8, \\ Zona Universitaria, 78290 San Luis Potosí, S.L.P.-México \\ (e-mail: jmartine@uaslp.mx, perezjor@uaslp.mx)
}

\section{Resumen}

Se analiza el efecto de la trayectoria académica en el desempeño de estudiantes de ingeniería en evaluaciones nacionales y se propone un modelo de predicción a partir del análisis estadístico del historial académico de alumnos de ingeniería en la Universidad de San Luis Potosí, México. Se realizó un estudio paramétrico de las variables mas significativas y se calibró el modelo a partir de la correlación con los resultados del examen nacional de egreso, alcanzando niveles de pronóstico del orden del $86 \%$ de la población estudiada. Se concluye en la utilidad de este tipo de herramientas analíticas para conducir a evaluaciones mas justas.

\section{Effect of History in the Academic Performance of Engineering Students in National Assessments}

\begin{abstract}
The effect of the academic background on the academic performance of engineering students in national tests is analyzed and a predictive model based on statistical analysis of the academic record of engineering students at the University of San Luis Potosi, Mexico, is proposed. A parametric study on the most significant parametric variables was conducted and the model was adjusted using the results of the national exam, reaching levels of predictions of the order of $86 \%$ of the population studied. It is concluded that this type of analyses are useful to obtain more adequate and fair evaluations.
\end{abstract}

Keywords: student performance, academic background, parametric analysis, engineering 


\section{INTRODUCCIÓN}

Indudablemente la educación superior constituye la puerta de acceso mas importante a la sociedad del conocimiento, toda vez que representa el medio ideal para el acrecentamiento del capital humano y de su inteligencia individual y colectiva; así fue reconocido en la Conferencia Mundial sobre Educación Superior organizada por la UNESCO en 1998: “...la educación superior y la investigación forman hoy en día la parte fundamental del desarrollo cultural, socioeconómico y ecológicamente sostenible de los individuos, las comunidades y las naciones" (UNESCO, 1998).

Existen abundantes opiniones respecto a los retos actuales a los que debe enfrentarse la educación en México y en particular el subsistema de educación superior; la Secretaría de Educación Pública del Gobierno Federal, a través de su plan sectorial 2007-2012 los ha resumido en tres grandes desafíos: cobertura con equidad, integración del sistema educativo y calidad (SEP, 2007). Aún cuando la equidad sigue siendo el mayor reto del Sistema Educativo Nacional y que durante el siglo pasado el gobierno federal dedicó la mayor parte de sus esfuerzos a la expansión de dicho sistema, lo cierto es que la calidad de los servicios educativos no ha sido atendida con la misma intensidad ni está a la altura del nivel educativo deseable para el país. La calidad de los servicios educativos debiera ser una aspiración constante compartida por el conjunto de la sociedad y uno de los principales objetivos de las reformas educativas en los países de Latinoamérica (OREALC/UNESCO, 2007).

Usualmente se considera que el concepto de calidad en educación debe ser asociado casi única y exclusivamente con el de evaluación académica, toda vez que la calidad requiere de un juicio valorativo que viene dado precisamente por la evaluación (Garduño, 1999), no obstante que esta, por sí sola, no mejora la calidad de un sistema. Sin embargo, los resultados obtenidos a través de instrumentos de evaluación pueden constituirse en un referente útil en la implementación de estrategias que contribuyan a mantener y elevar la calidad académica de la preparación de estudiantes de licenciatura; Alejandro Tiana señala la conveniencia de la evaluación en relación con la administración y gestión de los sistemas educativos como base para la toma de decisiones: "Sólo si un sistema educativo dispone de mecanismos estables y continuados de evaluación podrá producir informaciones útiles para alumbrar alternativas de modo rápido y fiable" (Tiana et al., 1996).

La evaluación del aprendizaje, si bien es un elemento inherente del proceso de enseñanza que puede ser considerado esencial a todo sistema educativo, tradicionalmente se ha aplicado en México de manera individual. El antecedente más remoto de la investigación y la medición educativa es el Instituto Nacional de Psicopedagogía creado en 1936, que sin embargo no desarrolló una actividad significativa (Martínez Rizo, 2001). En la década de 1960 comenzaron a aplicarse pruebas en gran escala para el ingreso a la UNAM, aunque ello no dejó de ser una excepción, y en la década siguiente la SEP inició la aplicación de pruebas estructuradas de aprendizaje a muestras nacionales de niños de primaria, pero sin cuidados técnicos para asegurar la equivalencia.

En las últimas décadas se ha observado un interés creciente por la evaluación del aprendizaje a gran escala, lo que ha permitido un mejor entendimiento y caracterización del logro educativo de los estudiantes. A partir de 1989 los sistemas nacionales de evaluación de calidad en educación superior registraron avances en varios frentes: en 1991 se crean los Comités Interinstitucionales de Evaluación de Educación Superior (CIEES), organismos encargados de la evaluación de programas académicos de licenciatura con un enfoque hacia la mejora permanente; en 1994 se crea el Centro Nacional de Evaluación A.C. (CENEVAL), cuyo objetivo se centra en la elaboración y aplicación de instrumentos de evaluación a nivel nacional para el ingreso a educación media superior, superior y posgrado, así como para el egreso de programas de licenciatura; ese mismo año de 1994 se protocoliza el Consejo de Acreditación de la Enseñanza de la Ingeniería (CACEI) entre cuyas funciones principales está el "contribuir al mejoramiento de la calidad del ejercicio profesional de la ingeniería" (Ocampo, 1994) mediante el establecimiento de sistemas de acreditación. 
El año 2000, a su vez, fue testigo de la creación del Consejo para la Acreditación de la Educación Superior (COPAES), asociación civil conformada por representantes del gobierno federal, de las instituciones de educación superior y de los colegios de profesionales en diversas áreas del conocimiento, cuya finalidad consiste en el reconocimiento de las agencias acreditadoras de los programas de educación superior. Todos estos organismos fueron creados con un solo objetivo, la búsqueda de la calidad en la Educación Superior teniendo como instrumento principal diversos procesos de evaluación diagnóstica (Martínez Rizo, 2001).

Los estudios encaminados a explorar la capacidad de los instrumentos de evaluación para determinar la probabilidad de éxito de quienes los sustentan son realmente escasos. Como ya se ha mencionado, desde hace más de 30 años la SEP, a través de la Dirección General de Evaluación, desarrolla proyectos específicos de evaluación del aprendizaje teniendo como objetivos fundamentales el establecimiento de sistemas de medición del logro educativo que puedan proporcionar periódicamente información válida para determinar su avance, estancamiento o retroceso y que permita correlacionar éste con otras variables, así como la prueba de modelos matemáticos para la equiparación de resultados en el tiempo, independientemente de las modificaciones curriculares (Velázquez, 1996).

En un estudio efectuado por Chaín y otros en 2002, a partir de información disponible de 6937 estudiantes de la Universidad Veracruzana, se analizaron las relaciones entre los resultados obtenidos en el Examen Nacional de Ingreso a la licenciatura y su trayectoria escolar, planteando un modelo que permite calcular la probabilidad de éxito escolar con relativa precisión (Chaín et al., 2003). De manera más extensiva se han realizado estudios encaminados a analizar el efecto de diversas variables de tipo contextual en el desempeño académico. Es indiscutible, por ejemplo, el efecto que ejercen el nivel socioeconómico y los factores familiares en el rendimiento académico, efecto que puede ser confirmado por diversas investigaciones en el ámbito internacional (Jones y White, 2000; Ruiz, 2001; González-Pienda et al., 2002; Casanova et al., 2005). Otra de las variables que mas inciden en el desempeño del alumno es el contexto del régimen de la escuela, efecto que ha sido reconocido en la mayor parte de la literatura sobre eficacia escolar (Carvallo, 2005). El efecto de otro tipo de variables tales como la edad del estudiante, el género, el profesor y el turno de estudios, ha sido analizados también por diversos autores (Carvallo et al., 2007), obteniendo resultados relevantes que permiten sostener o rechazar el grado de correlación existente con el logro educativo.

En este contexto, y con base en un análisis estadístico del historial académico de estudiantes de ingeniería sujetos a instrumentos nacionales de evaluación, en este trabajo se postula el desarrollo de un modelo que permite predecir, en orden inverso al que se ha planteado por la mayor parte de los estudios aquí referidos, los niveles de desempeño y grado de éxito esperados durante la consecución de dichas pruebas, en base al análisis de su trayectoria académica durante la licenciatura; el grado de correlación obtenido es razonablemente alto, permitiendo alcanzar niveles de pronóstico positivo en prácticamente el $86 \%$ de la población analizada. Aún reconociendo las limitaciones del estudio asociadas al tamaño y configuración de la muestra, este permite ampliar el conocimiento sobre el efecto del historial académico en el desempeño futuro de estudiantes de educación superior.

\section{CONFIABILIDAD DE LAS EVALUACIONES}

Los principales criterios a que deben atender los instrumentos de evaluación se refieren a los que tienen que ver con la confiabilidad y validez, entendiéndose el primero como la capacidad de la prueba para medir algo con precisión o en forma consistente, y el segundo como la demostración de que dicha prueba mide lo que se pretende medir. Un tipo de validez que es de gran importancia se refiere a la validez predictiva, la cual consiste en la determinación de la correlación que debe existir entre los resultados de una prueba y un determinado criterio de referencia, normalmente una actitud futura (Hernández Uralde, 2005). Un buen ejemplo de ello lo constituye el examen de admisión a la licenciatura para las carreras de ingeniería en la Universidad Autónoma de San Luis Potosí, el cual, a partir de la manipulación estadística de los datos históricos disponibles relativos al desempeño de los aspirantes en dicho examen, puede constituirse en un instrumento útil de 
predicción de su propia trayectoria académica ya como estudiantes de los diversos programas de ingeniería que oferta la Universidad en términos de permanencia y de eficiencia terminal.

Tomando como población de estudio los estudiantes de las cohortes que ingresaron al programa de Ingeniería Civil en la UASLP entre los años 1990 y 1996 (no es de utilidad en estos momentos las generaciones posteriores ya que aún cuentan con elementos en su etapa escolar). Si se colocan en orden ascendente los lugares obtenidos en dicha evaluación por los estudiantes de una misma cohorte, es deseable suponer que los $n$ primeros lugares de la lista serán quienes finalmente concluyan de manera satisfactoria sus estudios, siendo $n$ la eficiencia terminal de dicha cohorte; si ello sucede de esa manera, existirá un $100 \%$ de correlación entre los resultados del examen de admisión y la eficiencia de egreso de la cohorte, y aquél se convertiría en un instrumento útil para poder conocer de antemano quiénes de nuestros alumnos concluirán el programa y quienes abandonarán prematuramente. Si por el contrario, quienes concluyen satisfactoriamente el plan de estudios son los $n$ últimos aspirantes de la lista, no existirá absolutamente ninguna congruencia con los resultados de la admisión y la correlación será de 0 $\%$.

Llámese $A$, a la suma de los $n$ primero lugares de una serie aritmética ascendente del tamaño de una cohorte de nuevo ingreso, y llámese $B$ a la suma de los $n$ últimos lugares. Llámese $x$ a la suma de los lugares obtenidos en el examen de admisión por los $n$ alumnos que egresaron de dicha cohorte. Si las predicciones del examen fueran correctas, el valor de $x$ debería coincidir con el de A y si la correlación fuera nula debería coincidir con B. Valores intermedios pueden ser obtenidos mediante interpolación lineal entre estos extremos mediante una sencilla fórmula:

Índice de correlación $=\frac{B-x}{B-A} \times 100$

La Tabla 1 muestra los índices de correlación así obtenidos para los alumnos de primer ingreso en las cohortes generacionales 1990 a 1996 de Ingeniería Civil. El valor promedio de $69.3 \%$ indica el grado del poder de predicción del examen de admisión a la licenciatura de ingeniería civil de la UASLP para discriminar los estudiantes de una determinada cohorte generacional de primer ingreso que concluirán la totalidad del plan de estudios de quienes no lo harán; implica la probabilidad de que un aspirante a ingresar al programa, cuya calificación del examen de admisión lo ubique en los $\mathbf{n}$ primeros sitios (siendo $\mathbf{n}$ el número de alumnos que previsoramente concluirán la carrera con base en datos históricos) pueda efectivamente alcanzar dicha meta.

Tabla 1: Índices de Correlación Ingreso-Egreso, generaciones 1990 a 1996

\begin{tabular}{|c|c|}
\hline Cohorte Generacional & Índice de correlación \\
\hline 1990 & $65.8 \%$ \\
\hline 1991 & $69.7 \%$ \\
\hline 1992 & $75.3 \%$ \\
\hline 1993 & $65.9 \%$ \\
\hline 1994 & $69.1 \%$ \\
\hline 1995 & $71.8 \%$ \\
\hline 1996 & $67.6 \%$ \\
\hline Promedio & $69.3 \%$ \\
\hline
\end{tabular}

El examen de admisión para ingresar en cualquier licenciatura de ingeniería ofertada por la Universidad Autónoma de San Luis Potosí está compuesto de dos partes, la primera de las cuales, con un peso ponderado del $15 \%$, evalúa habilidades de aprendizaje, mientras que la segunda, mas extensa, califica el conocimiento adquirido por el estudiante durante su paso por el bachillerato en áreas como física, química y matemáticas. A pesar de ser una evaluación muy 
completa, esta adolece, al igual que la mayoría de los exámenes de admisión o selección en otra instituciones de educación superior, de un área que por su importancia recientemente se ha incorporado en gran parte en los perfiles de ingreso y egreso de los programas de licenciatura: la evaluación de las actitudes del estudiante de cara a los problemas que habrá de enfrentar en su vida laboral, y que de manera conjunta con los conocimientos y habilidades adquiridos constituyen el bagaje que lo convertirá en auténtico profesional. Es posible identificar en el fenómeno del rezago académico y la deserción una buena dosis de causas atribuibles a problemas que tienen que ver con la actitud del estudiante (Pérez González, 2006); sin ahondar demasiado en esta aseveración, aún cuando el poder de predicción de nuestro instrumento de evaluación es prácticamente de un $70 \%$, lo cierto es que de incorporar en su proceso un examen que valore las actitudes del aspirante (cuya ponderación en el resultado global deberá ser calibrada) seguramente este valor aumentará considerablemente convirtiéndose en un poderoso instrumento en la toma de decisiones para una eficaz y eventual implementación de programas de seguimiento individualizado de los estudiantes de menor rendimiento, incluso desde antes de su ingreso definitivo en la Institución.

\section{METODOLOGÍA DEL MODELO PROPUESTO}

De manera similar al concepto de validez predictiva de un instrumento de evaluación, en el cual se busca correlacionar, como ya se ha mencionado, los resultados de dicha prueba con una cierta actitud futura, es posible también predecir, en orden inverso, el resultado esperado del probable desempeño de egresados de programas de ingeniería sometidos a evaluaciones nacionales, a partir del análisis de su trayectoria académica durante la licenciatura mediante el empleo de modelos matemáticos que tomen en cuenta las variables mas significativas durante dicho proceso.

Para la elaboración del modelo predictivo se consideraron los resultados de los sustentantes del Examen General de Egreso de la Licenciatura en Ingeniería Civil (EGEL-IC) que diseña, elabora y aplica el CENEVAL, instrumento de cobertura nacional que mide y evalúa los conocimientos y aptitudes que son resultado de la formación académica de los recién egresados de los programas de Ingeniería Civil.

\section{Población de estudio}

La población de análisis la constituye un total de 178 jóvenes egresados de ingeniería de la Universidad Autónoma de San Luis Potosí que han sustentado el examen desde su creación en octubre de 1996 hasta octubre de 2007; esta muestra incluye alumnos de las cohortes que ingresaron entre los años de 1989 a 2002. El grupo representa una población totalmente aleatoria toda vez que la aplicación del examen es voluntaria y no existe intervención alguna para su selección, además de la heterogeneidad de su desempeño académico a lo largo de la licenciatura, tomando como referencia factores como promedio general, cantidad de materias reprobadas, rendimiento, etc. Por todo lo anterior se concluye que dicha población constituye una muestra adecuada para que las determinaciones que se infieran de su análisis tengan una alta confiabilidad.

El resultado global del sustentante de la prueba en cuestión se expresa en una escala especial denominada Índice CENEVAL, cuyo rango va de 700 a 1300 puntos, correspondiendo estos con una calificación de 0 a $100 \%$ respectivamente, con un dominio satisfactorio de 1000 a 1149 puntos y un alto rendimiento de 1150 a 1300 (a quienes obtienen el dominio satisfactorio se les otorga un Testimonio de Desempeño Satisfactorio o TDS, mientras que los que alcanzan el dominio sobresaliente se hacen acreedores a un Testimonio de Desempeño Sobresaliente o TDSS). De la totalidad de la muestra, 109 individuos que constituyen el $61.2 \%$ alcanzó el dominio satisfactorio (arriba de 1000 puntos en el índice CENEVAL), mientras que 24 de ellos, que representan el $13.5 \%$, el dominio sobresaliente. Por el número de sustentantes, este programa de ingeniería civil se ubica entre los que mas han aportado a nivel nacional, aún cuando el examen no es obligatorio. 


\section{Análisis paramétrico}

Se usaron seis parámetros relacionados con la trayectoria académica que fueron considerados para la determinación del modelo de pronóstico, así como un breve análisis de su correlación con los resultados obtenidos en el examen general, incluyendo un juicio de valor meramente cualitativo:

\section{1) Lugar obtenido en el examen de admisión a la licenciatura (P1).}

Este parámetro oscila entre 1 y 700 con una media de 227. El análisis muestra que los sustentantes del EGEL que obtuvieron TDSS lograron un lugar promedio en el examen de admisión de 139.2, los que se hicieron acreedores a un TDS un lugar promedio de 206.6, mientras que aquellos que no alcanzaron los 1000 puntos obtuvieron en promedio el lugar número 311.8, lo que permite establecer que este parámetro es medianamente significativo como antecedente para estimar su desempeño en el EGEL.

\section{2) Promedio general de calificaciones obtenido durante los estudios de licenciatura (P2).}

Los sustentantes que obtuvieron TDSS Iograron un promedio general en su conjunto durante la licenciatura de 77.2, quienes obtuvieron TDS tuvieron un promedio general en conjunto de 70.9 y los que no lograron alcanzar los 1000 puntos obtuvieron en conjunto un promedio general de 67.9; además el 90\% de los alumnos que obtuvieron un promedio general menor a 65.0 no alcanzó una puntuación superior a 1000. Lo anterior permite concluir la alta correlación de este parámetro en nuestro modelo predictivo.

\section{3) Promedio aprobatorio de calificaciones obtenido en la licenciatura (P3).}

El análisis de correlación respectiva muestra que los alumnos que obtuvieron TDSS en el EGEL lograron un promedio aprobatorio en conjunto en la licenciatura de 80.5, quienes obtuvieron TDS alcanzaron un promedio de 76.1 y aquellos que no alcanzaron el índice de 1000 puntos obtuvieron un promedio aprobatorio durante la licenciatura de 75.2 en su conjunto, mostrando una considerable correlación de este parámetro con los resultados que un alumno puede obtener en dicho examen.

\section{4) Número de semestres en que el sustentante cursó el programa de licenciatura (P4).}

Este parámetro no tiene un efecto determinante sobre la estimación de los resultados que el sustentante puede obtener en el Examen General de Egreso. Lo anterior se concluye al observar que alumnos que cursaron su carrera profesional en ocho semestres no lograron una puntuación satisfactoria en el examen, mientras que algunos egresados que concluyeron el plan de estudios hasta en dieciséis semestres se encuentran entre los que obtuvieron un desempeño sobresaliente. Por otro lado, no existen grandes diferencias en los resultados alcanzados en los tres grupos ya que los sustentantes que obtuvieron TDSS cursaron su carrera en 10.5 semestres en promedio, mientras que aquellos que obtuvieron un desempeño satisfactorio lo hicieron en un promedio de 10.8 semestres y los que no alcanzaron los 1000 puntos tardaron en promedio 11.5 semestres en concluir sus estudios de licenciatura.

\section{5) Rendimiento académico durante el curso de la licenciatura (P5).}

El rango de variación de este parámetro para los sustentantes de la población bajo estudio oscila entre $41.2 \%$ y $100 \%$, el cual como se ve es bastante amplio. Así, el grupo de alumnos que obtuvieron el TDSS logró durante su estancia en la Facultad un rendimiento promedio de 78.3\%, mientras que aquellos que tan solo obtuvieron un desempeño satisfactorio lograron en su conjunto un rendimiento promedio de $67.8 \%$ y los que no alcanzaron el índice de 1000 puntos en el EGEL observaron un rendimiento combinado de $61.7 \%$; por lo tanto, este parámetro es uno de los que mayor significancia tiene como antecedente para estimar el comportamiento de un egresado en dicho examen.

6) Número de materias reprobadas por el sustentante durante su estancia en la Facultad (P6). 
Este parámetro oscila entre 0 y 20 materias reprobadas. Si bien algún alumno con un número de reprobadas considerable logró un desempeño satisfactorio, ninguno de ellos tuvo un desempeño sobresaliente. Observamos que el grupo de sustentantes que lograron el TDSS reprobó en promedio 2.7 materias durante la licenciatura mientras que los que alcanzaron la puntuación correspondiente al TDS tuvieron en promedio 5.1 materias reprobadas; el resto reprobaron en su conjunto un promedio de 8.0 materias, por lo que se deduce una fuerte correlación entre este parámetro y la posibilidad de lograr un buen resultado en el Examen General de Egreso.

\section{7) Número de exámenes de regularización presentados por el sustentante (P7).}

Este parámetro puede alcanzar valores hasta de 10 exámenes de regularización presentados en el transcurso de la licenciatura. El análisis por percentiles indica que mas del $62 \%$ de los sustentantes no presentaron ningún examen de regularización en el transcurso de su carrera, y tan solo el $14.2 \%$ presentó tres o mas exámenes de este tipo. Al observar los resultados obtenidos en el EGEL por el grupo de sustentantes que constituyen la población de estudio, se observa que este parámetro tiene un efecto no determinante en su correlación con dicho desempeño. La Tabla 2 resume los parámetros arriba analizados

En base a lo anterior, en la Tabla 3 se puede observar la manera cómo se incorporará al modelo predictivo cada una de las variables tratadas en los párrafos anteriores, donde los valores de las constantes de ponderación $W_{1}, W_{2}, \ldots W_{7}$, deberán ser ajustados en función de los resultados obtenidos.

Tabla 2: Resumen de parámetros empleados en el modelo predictivo

\begin{tabular}{|l|c|c|c|c|c|c|c|c|}
\hline $\begin{array}{c}\text { Grupo de } \\
\text { Sustentantes }\end{array}$ & P1 & P2 & P3 & P4 & P5 & P6 & P7 & $\begin{array}{c}\text { Calif. } \\
\text { CENEVAL }\end{array}$ \\
\hline $\begin{array}{l}\text { Sustentantes con } \\
\text { TDSS }\end{array}$ & 139.2 & 77.2 & 80.5 & 10.5 & $78.3 \%$ & 2.7 & 0.3 & 1188 \\
\hline $\begin{array}{l}\text { Sustentantes con } \\
\text { TDS }\end{array}$ & 206.6 & 70.9 & 76.1 & 10.8 & $67.8 \%$ & 5.1 & 0.9 & 1046 \\
\hline $\begin{array}{l}\text { Sustentantes con } \\
\text { menos de 1000 } \\
\text { puntos }\end{array}$ & 311.8 & 67.9 & 75.2 & 11.5 & $61.7 \%$ & 8.0 & 1.8 & 958 \\
\hline $\begin{array}{c}\text { Resultados } \\
\text { Globales }\end{array}$ & 227.0 & 71.7 & 77.4 & 11.1 & $68.0 \%$ & 5.8 & 1.1 & 1049.7 \\
\hline
\end{tabular}

Tabla 3: Manejo matemático de los parámetros de desempeño académico

\begin{tabular}{|l|l|c|c|}
\hline Variable & Descripción de la variable & Clave de la variable & $\begin{array}{c}\text { Manejo matemático } \\
\text { de la variable }\end{array}$ \\
\hline P1 & $\begin{array}{l}\text { Lugar ocupado en el examen } \\
\text { de admisión }\end{array}$ & $\mathrm{X}_{1}$ & $\left(\left(700-\mathrm{X}_{1}\right) / 700\right)^{\star}\left(\mathrm{W}_{1}\right)$ \\
\hline P2 & Promedio general & $\mathrm{X}_{2}$ & $\left(\mathrm{X}_{2} / 100\right)^{\star}\left(\mathrm{W}_{2}\right)$ \\
\hline P3 & Promedio aprobatorio & $\mathrm{X}_{3}$ & $\left(\mathrm{X}_{3} / 100\right)^{\star}\left(\mathrm{W}_{3}\right)$ \\
\hline P4 & $\begin{array}{l}\text { Cantidad de semestres } \\
\text { en que cursó la licenciatura }\end{array}$ & $\mathrm{X}_{4}$ & $\left(10 / \mathrm{X}_{4}\right)^{\star}\left(\mathrm{W}_{4}\right)$ \\
\hline P5 & Rendimiento académico & $\mathrm{X}_{5}$ & $\left(\mathrm{X}_{5} / 100\right)^{*}\left(\mathrm{~W}_{5}\right)$ \\
\hline P6 & $\begin{array}{l}\text { Cantidad de materias } \\
\text { reprobadas en la licenciatura }\end{array}$ & $\mathrm{X}_{6}$ & $\left(\left(20-\mathrm{X}_{6}\right) / 20\right)^{*}\left(\mathrm{~W}_{6}\right)$ \\
\hline P7 & Exámenes de regularización & $\mathrm{X}_{7}$ & $\left(\left(10-\mathrm{X}_{7}\right) / 10\right)^{*}\left(\mathrm{~W}_{7}\right)$ \\
\hline
\end{tabular}

Todos estos parámetros relacionados con la trayectoria académica de la población durante sus estudios de licenciatura, muestran en mayor o menor medida una correlación con su éxito o 
fracaso observado en la aplicación del Examen General de Egreso, por lo que son susceptibles de ser incorporados en un eventual modelo predictivo, al que se le denominará índice de Desempeño, mediante un adecuado manejo de su valor que tome en cuenta el rango de variación de dicho parámetro, como se ha indicado en la Tabla 3, así como un nivel de ponderación debidamente calibrado una vez aplicado el modelo a la población de estudio. De esta manera, el Índice de Desempeño pronosticado de un sustentante durante la aplicación del Examen General de Egreso de la Licenciatura estará dado por la expresión:

$$
\begin{aligned}
& \left(\left(700-\mathrm{X}_{1}\right) / 700\right)^{*}\left(\mathrm{~W}_{1}\right)+\left(\mathrm{X}_{2} / 100\right)^{*}\left(\mathrm{~W}_{2}\right)+\left(\mathrm{X}_{3} / 100\right)^{\star}\left(\mathrm{W}_{3}\right)+\left(10 / \mathrm{X}_{4}\right)^{\star}\left(\mathrm{W}_{4}\right) \\
& \quad+\left(\mathrm{X}_{5} / 100\right)^{*}\left(\mathrm{~W}_{5}\right)+\left(\left(20-\mathrm{X}_{6}\right) / 20\right)^{\star}\left(\mathrm{W}_{6}\right)+\left(\left(10-\mathrm{X}_{7}\right) / 10\right)^{*}\left(\mathrm{~W}_{7}\right)
\end{aligned}
$$

\section{RESULTADOS Y DISCUSIÓN}

El análisis de los parámetros de la historia académica de los sustentantes y su correlación con el desempeño alcanzado por los mismos en el EGEL, muestra que aquellos más fuertemente asociados con una probabilidad de éxito corresponden al promedio general alcanzado en la licenciatura $\left(X_{2}\right)$, su rendimiento académico durante la misma $\left(X_{5}\right)$ y la cantidad de materias que fueron reprobadas $\left(X_{6}\right)$, a los cuales fueron asignadas constantes de ponderación de 0.20, 0.25 y 0.20 respectivamente. De igual manera, los parámetros que de manera cualitativa muestran una regular correlación con las posibilidades del sustentante para obtener un alto resultado en el examen son aquellos correspondientes con el promedio aprobatorio $\left(\mathrm{X}_{3}\right)$, el lugar obtenido en el examen de admisión a la licenciatura $\left(X_{1}\right)$, así como la cantidad de exámenes de regularización presentados $\left(\mathrm{x}_{7}\right)$; a cada uno de ellos se les asignó una constante de ponderación de 0.10.

La variable relativa a la cantidad de semestres en que fue cursada la licenciatura $\left(\mathrm{X}_{4}\right)$ fue la que menos influyó en los resultados del EGEL, sin embargo también se incorporó al modelo de pronóstico, aunque con una tasa de ponderación muy pequeña, del orden de 0.05.

La expresión postulada para definir el Índice de Desempeño pronosticado puede ser rescrita, una vez incorporados los valores calibrados para las constantes de ponderación, de la siguiente manera:

$$
\begin{aligned}
& \left(\left(700-X_{1}\right) / 700\right)^{\star}(0.10)+\left(X_{2} / 100\right)^{\star}(0.20)+\left(X_{3} / 100\right)^{\star}(0.10)+\left(10 / X_{4}\right)^{\star}(0.05) \\
& +\left(X_{5} / 100\right)^{\star}(0.25)+\left(\left(20-X_{6}\right) / 20\right)^{\star}(0.20)+\left(\left(10-X_{7}\right) / 10\right)^{\star}(0.10)
\end{aligned}
$$

El dominio de aplicabilidad para la expresión anterior es de 0 a 100\% y se ha establecido como límite de referencia un valor por encima de $70 \%$ para correlacionar dicho parámetro con un desempeño satisfactorio en el Examen General de Egreso; ello significa que un sustentante cuyo índice de desempeño obtenido de la manera aquí expuesta, considerando su historial académico durante sus estudios de licenciatura, haya sido superior a $70 \%$, necesariamente deberá corresponder con un resultado satisfactorio en la prueba nacional, obteniendo un índice CENEVAL de al menos 1000 puntos.

Al aplicar la expresión dada para pronosticar el desempeño de los 178 sustentantes del EGEL que conforman la población de estudio, se encontró que 145 de ellos que corresponden al 81.4\% obtuvieron un pronóstico acertado al correlacionar de manera satisfactoria los resultados alcanzados en el examen con el Índice de Desempeño aquí descrito, tanto los que obtuvieron calificaciones arriba de 1000 puntos en el índice CENEVAL como los que estuvieron por debajo de este valor. 21 individuos más, representando un $11.8 \%$ de la muestra, obtuvieron valores muy cercanos al 70\%, por lo que pueden ser considerados como errores de pronóstico muy leves. El $6.8 \%$ restante, tan solo 12 de los sustentantes, puede ser considerado como errores considerables del modelo de pronóstico (fig. 1).

Por todo lo anterior, es posible concluir que el modelo hasta aquí esbozado cuenta con un margen de confiabilidad cercano al $93 \%$. 


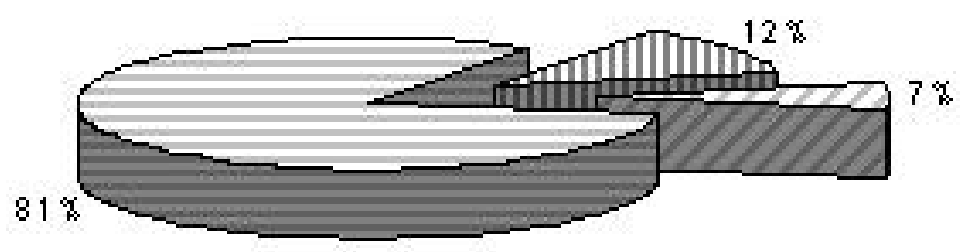

Fig. 1: Confiabilidad del modelo de pronóstico: $81 \%$ acierto, $12 \%$ error leve, $7 \%$ error grave

\section{CONCLUSIONES}

A partir de los resultados mostrados, de su análisis y discusión, es posible establecer un modelo de pronóstico que permita predecir con un razonable grado de precisión, el grado de éxito o fracaso de sustentantes de exámenes nacionales de egreso de la licenciatura en ingeniería, en base a su trayectoria académica. Se ha encontrado que las variables relacionadas con dicha trayectoria que mayor efecto tienen en la estimación de dicho desempeño corresponden al promedio general alcanzado en la licenciatura, el rendimiento académico y la cantidad de materias que fueron reprobadas; variables con menor grado de correlación dependen del promedio aprobatorio, del lugar obtenido en el examen de admisión y de la cantidad de exámenes de regularización presentados, y en mucho menor medida del tiempo de duración de los estudios de licenciatura.

Mediante un adecuado manejo de dichas variables que incluya una ponderación debidamente calibrada con el análisis estadístico de poblaciones representativas, es posible establecer modelos sencillos que permitan pronosticar de manera confiable el desempeño a futuro de cohortes de estudiantes de ingeniería, con la posibilidad incluso, de anticipar con un alto grado de precisión su propio desempeño profesional, materializándose así la posibilidad de ir un paso adelante en la instrumentación de acciones que contribuyan a mantener y elevar la calidad académica de su preparación y la posibilidad de establecer mecanismos mas justos de evaluación del aprendizaje.

\section{REFERENCIAS}

Carvallo, M.; Análisis de los resultados obtenidos en estudios de eficacia escolar en México comparados con los de otros países, Revista Electrónica Iberoamericana sobre Calidad, Eficacia y Cambio en Educación, ISSN: 1696-4713 (en línea) 3(2), 80-108, 2005. http://www.rinace.net/arts/ vol3num2/art6.htm. Acceso: Noviembre 2007.

Carvallo, M., J. Caso y L.A. Contreras; Estimación del efecto de variables contextuales en el logro académico de estudiantes de Baja California, Revista Electrónica de Investigación Educativa, ISSN: 1607-4041 (en línea) 9(2), 2007. http://redie.uabc.mx/vol9no2/contenido-carvallo.html. Acceso: Noviembre 2007.

Casanova, P.; M. Cruz, M.J. De la Torre y M. De la Villa; Influence of family and sociodemographic variables on students with low academic achievement, Educational Psychology, 25(4), 423-435 (2005).

Chaín, R., N. Cruz, M. Martínez y N. Jácome; Examen de selección y trayectoria escolar, Revista de la Educación Superior, ISSN: 0185-2760 (en línea) XXXII(125), enero-marzo 2003. http://www.anuies.mx/servicios/p_anuies/index2.php?clave=publicaciones/revsup/res125/art2.htm. Acceso: Noviembre 2007. 
Garduño, L.R.; Hacia un modelo de evaluación de la calidad de instituciones de educación superior, Revista Iberoamericana de Educación, ISSN: 1681-5653 (en línea) I(21), septiembrediciembre 1999. http://www.rieoei.org/rie21f.htm. Acceso: Noviembre 2007.

González-Pienda, y otros cinco autores; A structural equation model of parental involvement, motivational and aptitudinal characteristics and academic achievement, The Journal of Experimental Education, 70(3), 257-287 (2002).

Hernández Uralde, J.; Validez y confiabilidad en las pruebas de aprendizaje, Boletín Ceneval, 1(13), 1-4 (2005).

Jones, I. y S. White; Family composition parent involvement and young children's academic Achievement, Early Child Development and Care, (161), 71-82 (2000).

Martínez Rizo, F.; Federalización y subsistemas estatales de educación superior. Las políticas educativas mexicanas en los años noventa, Revista Mexicana de Investigación Educativa, 6(13), 425-453 (2001).

Martínez Rizo, F.; Evaluación educativa y pruebas estandarizadas. Elementos para enriquecer el debate, Revista de la Educación Superior, ISSN: 0185-2760 (en línea) XXX (120), octubrediciembre 2001. http://www.anuies.mx/servicios/p_anuies/index2.php?clave=publicaciones/revsup/res120/art5.

htm. Acceso: Noviembre 2007.

Ocampo, J.F.; La acreditación y su relación con la calidad de la enseñanza, Revista Ingeniería Civil, (307), 10-12 (1994).

OREALC/UNESCO; Organización de las Naciones Unidas para la Educación, la Ciencia y la Cultura, Oficina Regional de Educación para América Latina y el Caribe; El derecho a una educación de calidad para todos en América Latina y el Caribe, Revista Electrónica Iberoamericana sobre Calidad, Eficacia y Cambio en Educación, ISSN: 1696-4713 (en línea) 5(3), 1-21, 2007. http://www.rinace.net/ arts/vol5num3/art1.pdf. Acceso: Noviembre 2007.

Pérez González, J.A; La Eficiencia Terminal en Programas de Licenciatura y su Relación con la Calidad Educativa, Revista Electrónica Iberoamericana sobre Calidad, Eficacia y Cambio en Educación, ISSN: 1696-4713 (en línea), 4(1), 2006. http://www.rinace.net/arts/vol4num1/art9.pdf. Acceso: Octubre 2007.

Ruiz, C.; Factores familiares vinculados al bajo rendimiento, Revista Complutense de Educación, 12(1), 81-113 (2001).

SEP; Secretaría de Educación Pública, Gobierno de los Estados Unidos Mexicanos; Programa Sectorial de Educación 2007-2012, $1^{\text {a }}$ edición, 9-22. Comisión Nacional de Libros de Texto Gratuitos, México, D.F. (2007).

Tiana, A., H. Santángelo y F. Pedró; Evaluación de la calidad de la educación, Revista Iberoamericana de Educación, ISSN: 1681-5653 (en línea) I(10), enero-abril 1996. http://www.rieoei.org /oeivirt/rie10a09.htm. Acceso: Noviembre 2007.

UNESCO; Organización de las Naciones Unidas para la Educación, la Ciencia y la Cultura; Declaración Mundial sobre la Educación Superior en el Siglo XXI: Visión y Acción, Revista de la Edu-cación Superior, ISSN: 0185-2760 (en línea) XXVII(107) julio-septiembre 1998. http://www.anuies.mx /servicios/p_anuies/publicaciones/revsup/res107/art4.htm. Acceso: Octubre 2007.

Velázquez, V.M.; La evaluación como recurso para elevar la calidad de la educación en México, Revista Iberoamericana de Educación, ISSN: 1681-5653 (en línea) I (10), enero-abril 1996. http://www.rieoei.org/oeivirt/rie10a08.htm. Acceso: Noviembre 2007. 